\title{
Favorable outcome of allogenic hematopoietic stem cell transplantation in farber disease
}

\begin{abstract}
Farber disease is a rare autosomal recessively inherited disorder caused by acid ceramidase deficiency. Painful subcutaneous skin nodules, progressive joint stiffness, and laryngeal involvement leading to a progressive hoarseness are the main characteristics of the disease. We report favorable outcome of hematopoietic stem cell transplantation after 24 months of follow up in a 2.7-years-old girl with Farber's disease who was transplanted from her 49-years-old maternal grandfather resulting in resolution of her symptoms. Hematopoetic stem cell transplantation could provide sufficient amount of enzyme and accordingly diminish or stabilize the symptoms in these patients.
\end{abstract}

Keywords: farber disease $\cdot$ hematopoietic stem cell transplantation $•$ central nervous system

\section{Introduction}

Disseminated lipogranulomatosis, known as 'Farber disease' (FD; OMIM 228000), is a rare autosomal recessively inherited disorder, which is caused by mutations in both alleles of the ASAH1 gene, resulting in acid ceramidase deficiency and accumulation of inflammatory sphingolipid, ceramide [1,2]. Painful subcutaneous skin nodules, typicallyin proximity to the joints, most often interphalangeal, wrist, elbow and ankle joints, or over points of mechanical pressure are the characteristic clinical presentation of the disease.Progressive joint stiffness, limitation of motion by contractures and finally immobilization and deformation of joints usually follows the mentioned signs and symptoms. In addition, laryngeal involvement could lead to a progressive hoarseness [3].

Seven phenotypes differing in severity and organ involvement including lungs, nervous system, heart and lymph nodes have been described [4]. The degree of Central Nervous System (CNS) involvement which leads to progressive neurologic deterioration depends on residual lysosomal ceramidase turnover [5]. Pulmonary disease with interstitial pneumonia could also result in patients' death. Most patients present symptoms between the newborn period and the first birthday. Mortality mostly happens within the first years of life, but prolonged courses in patients without severe CNS disease may also be observed [6]. However, in children without CNS involvement, granulomatous inflammation still causes a disabling course. Children become wheel chair dependent due to progressive joint deformities in early infancy. Chronic respiratory failure as a result of granulomatous inflammation of the lower airways occurs and death typically comes before 20 years of age.

Clinical diagnosis of the disease could be confirmed by determination of acid ceramidase activity measured in cultured skin fibroblasts, white blood cells or amniocytes. Demonstration of granulomas with macrophages containing lipid cytoplasmic inclusions in biopsy specimen of subcutaneous nodules or other tissues is another diagnostic approach. Chromatography or mass spectrometry could also determine the ceramide accumulation in tissues [7].

The available treatment for FD is symptomatic aiming to reduce pain and inflammation. Enzyme Replacement Therapy (ERT) in which the recombinant protein is introduced into patients by intravenous infusions is currently available for 7 lysosomal storage disorders, and under evaluation for several others [8]. Although ERTs have proven to be safe and the recombinant enzymes cannot cross the blood-brain barrier, owing to the size of the molecule and therefore have little or no effect in CNS involvements [9]. Lentiviral gene therapy is being explored in animal models. For some decades now, allogeneic Hematopoeitic Stem Cell Transplantation (HSCT) has shown efficacy in some inherited disorders including lysosomal storage disorders [10]. Since the lack of enzyme activity causes the symptoms of these metabolic disorders, transplantation of hematopoetic stem cells from a healthy donor could provide sufficient amount of enzyme and accordingly diminish or stabilize the symptoms [11].

\section{Amir Ali Hamidieh, Tahereh Rostami*, Maryam Behfar, Azadeh Kiumarsi \& Ardeshir Ghavamzadeh}

Department of Hematology-Oncology, Tehran University of Medical Science, Iran

*Author for correspondence:

trostami@sina.tums.ac.ir 
Moreover, in FD, a dysregulation of leukocyte functions, probably due to the intracellular role of ceramide in intracellular signal transduction adds to the curative effect of HSCT. In the present case with FD, we report the sustained reversal ofceramide accumulation and granulomatous infiltration following allogeneic HSCT.

\section{Case report}

A 2.7-years-old girl with Farber's disease from consanguinous parents reffered to Hematology-Oncology and SCT Research Center (HORCSCT). She presented at the age of 11 months with characteristic features of FD including hoarseness, multiple subcutaneous skin nodules and decreased range of motion of digital joints. Diagnosis of the disease was confirmed by genetic assessment that was homozygote mutation for c. $830 \mathrm{c}>$ Ain exon 11. Cranial MRI and spiral chest CT scan were normal and no other organ involvement was found at that time. Although no sibling were available, her 49 years old maternal grandfather who was heterozygote for the mentioned mutation but was fully (10/10) antigen human leukocyte antigen (HLA)-matched offered his Bone Marrow stem cells $(1.7 \times 106 \mathrm{CD} 34+$ cells $/ \mathrm{kg}$ recipient body wt) and HSCT was therefore performed using a myeloablative conditioning regimen comprising busulfan (12.8 $\mathrm{mg} / \mathrm{kg})$, cyclophosphamide $(200 \mathrm{mg} / \mathrm{kg})$ and antithymocyte globulin (Thymoglobulin). GvHD prophylaxis consisted of standard methotrexate and cyclosporine. Neutrophil engraftment occurred on day +13 and she had a stable graft with $95 \%$ donor chimerism by STR-PCR on day +15 .

After transplantation, complications included mucositis (CTC grade 2), grade I GvHD of the skin (stage II) that responded to a short course of prednisolone, and CMV reactivation (by PCR) which underwent preemptive therapy with Ganciclovir. She was discharged from the hospital on day +52 . Her characteristic hoarseness disappeared gradually during the first year after HSCT, and, most importantly, her joint mobility improved within the second year after transplantation. She is now 24 months past transplantation, is growing well and she walks and speaks normally. The graft is stable, with a chimerism of $95 \%$ donor cells, as determined by PCR analysis of Short Tandem Repeat-markers (STR-PCR).

\section{Discussion}

The underlying theory for using allogeneic cell transplantation in FD is that the donorderived leukocytes would take part in enzyme delivery and metabolic correction [12]. The first reports on HSCT in FD was on patients who had a subtype with involvement of the CNS in whom transplantation did not seem to provide a benefit for these children, as it was not able to abolish or even reduce the neurotoxic effects of excessive ceramide accumulation in the brain [13]. There are even published such cases with partial initial therapeutic response but ongoing neurological progression [14]. However, as HSCT can provide a healthy immune system and thus correct the abnormal inflammatory response, it became a treatment option in those children with a predominance of the inflammatory component. Resolution of granulomas after transplantationhas also been demonstrated in children with CNS involvement [15].

In a new published cohort study of ten transplanted FD patients from Europe, the overall survival was $80 \%$ and the inflammatory symptoms in all patients resolved within a few weeks post-transplant [16].

As no unified data base for FD exists around the world, an ongoing case reporting of this ultra-rare disease will contribute to the available information and hopefully lead to achieving better results in these patients. Generally, based on the existing case reports, HSCT appears to be beneficial for the peripheral manifestations but the CNS manifestations may benefit from very early transplantation and in severe neurological cases HSCT may be acceptable as amelioration of other manifestations may improve their quality of life.

\section{References}

1. Ehlert K, Frosch M, Fehse $\mathrm{N}$ et al. Farber disease: Clinical presentation, pathogenesis and a new approach to treatment. Pediatr. Rheumatol. 5(1):15 (2007).

2. Solyom A, Nwosu EO, Mitchell J et al. Farber disease (acid ceramidase deficiency) epidemiology: Literature review and patient cohort data indicate moderate and attenuated phenotypes are likely underrepresented in the medical literature and are underdiagnosed. Mol. Genet. Metab. 120(1), S124-S125 (2017).

3. Alves MQ, Le Trionnaire E, Ribeiro I et al. Molecular basis of acid ceramidase deficiency in a neonatal form of Farber disease: Identification of the first large deletion in ASAH1 gene. Mol. Genet. Metab. 109(3), 276-281 (2013).

4. Moser HW, Linke T, Fensom AH et al. Acid 
ceramidase deficiency: Farber lipogranulomatosis. In the metabolic and molecular bases of inherited disease 8th edition. 3573-3585 (2001).

5. Levade T, Moser HW, Fensom $\mathrm{AH}$ et al. Neurodegenerative course in ceramidase deficiency (Farber disease) correlates with the residual lysosomal ceramide turnover in cultured living patient cells. J. Neurol. Sci. 134(1-2), 108114 (1995).

6. Pavone L, Moser HW, Mollica F et al. Farber's lipogranulomatosis: Ceramidase deficiency and prolonged survival in three relatives. Johns. Hopkins. Med. J. 147, 193-196 (1980).

7. Farber S, Cohen J, Uzman LL. Lipogranulomatosis: A new lipoglycoprotein storage disease. J. Mt. Sinai. Hospital. 24(6), 816-837 (1957).

8. Desnick RJ, Schuchman EH. Enzyme replacement therapy for lysosomal storage diseases: Lessons from 20 years of experience and remaining challenges. Annu. Rev. Genomics Hum. Genet. 13, 307-335 (2012).

9. Zielonka M, Garbade SF, Kölker S et al. A crosssectional quantitative analysis of the natural history of Farber disease: an ultra-orphan condition with rheumatologic and neurological cardinal disease features. [Genet. Med. 20(5), 524 (2018).
10. Malatack JJ, Consolini DM, Bayever E. The status of hematopoietic stem cell transplantation in lysosomal storage disease. Pediatr. Neurol. 29(5), 391-403 (2003).

11. Wilcox WR. Lysosomal storage disorders: The need for better pediatric recognition and comprehensive care. J. Pediatr. 144, S3-S14 (2004).

12. Wynn RF, Wraith JE, Mercer J et al. Improved metabolic correction in patients with lysosomal storage disease treated with hematopoietic stem cell transplant compared with enzyme replacement. J. Pediatr. 154(4), 609-611 (2009).

13. Souillet G, Guibaud P, Fensom AH et al. Outcome of displacement bone marrow transplantation in Farber's disease: a report of a case. In Correction of certain genetic diseases by transplantation.137-141 (1989).

14. Cappellari AM, Torcoletti M, Triulzi $\mathrm{F}$ et al. Nervous system involvement in Farber disease. $J$. Inherit. Metab. Dis. 39, 149-150 (2016).

15. Yeager AM, Armfield-Uhas K, Coles CD et al. Bone marrow transplantation for infantile ceramidase deficiency (Farber disease). Bone. Marrow. Transplant. 26, 357-363 (2000).

16. Ehlert K, Levade T, Di Rocco M et al. Allogeneic hematopoietic cell transplantation in Farber disease. J. Inherit. Metab. Dis. Mar 29, 1-8 (2018). 